\title{
Is a nutritional therapeutic approach unsuitable for metabolically healthy but obese women?
}

\section{G. Perseghin}

Published online: 26 June 2008

(C) Springer-Verlag 2008

Keywords Hypocaloric diet - Hypoenergetic diet . Insulin resistance $\cdot$ Low-calorie diet - Low-energy diet . Obesity $\cdot$ Physical exercise

Metabolically healthy but obese postmenopausal women are a subset of obese individuals who do not show obesityrelated metabolic abnormalities such as insulin resistance, a pro-atherosclerotic lipid profile, a proinflammatory state and hypertension $[1,2]$. In longitudinal studies, this phenotype was associated with reduced risks of developing type 2 diabetes and cardiovascular disease [3].

These 'fit-fat individuals' are of interest because they constitute a model that may provide insight into the pathogenesis of insulin resistance in humans. In addition, they represent a potential clinical challenge in that they may require a different therapeutic strategy from that used to treat obese and insulin-resistant women.

With respect to the pathogenic issue, it is presently unclear why these women are protected. Initially, investigators focused on elucidating the mechanism underlying this protective effect, with the aim of identifying potential therapeutic targets for the treatment of obese and insulin-

\footnotetext{
G. Perseghin

Faculty of Exercise Sciences,

Center 'Physical exercise for health and wellness',

Università degli Studi di Milano,

Milan, Italy

G. Perseghin $(\bowtie)$

Internal Medicine-Section of Nutrition/Metabolism, Istituto Scientifico San Raffaele,

via Olgettina 60,

20132 Milan, Italy

e-mail: perseghin.gianluca@hsr.it
}

resistant individuals. It was reported that a lower visceral fat content, despite a high body fat content, and a lower accumulation of fat within ectopic sites may contribute to the favourable metabolic profile $[2,4]$. These metabolically healthy but obese postmenopausal women had lower circulating levels of high-sensitivity C-reactive protein compared with 'at-risk' women [5], suggesting that the lack of a systemic proinflammatory condition, common in obesity, may explain the protection. It is also possible that the higher insulin sensitivity may be due to a primary genetic metabolic event that contributes to a protective metabolic profile but at the same time influences the early onset of obesity. This is consistent with a study on Pima Indians showing that insulin sensitivity is a predictor of future obesity [6]. The importance of these distinctive features is confirmed by the profile of metabolically obese but normal-weight women, who are characterised by a diametrically opposite profile. These women have a higher body fat content in spite of a normal BMI, and show the cluster of phenotypic risk factors associated with the insulin resistance syndrome $[7,8]$.

With respect to the clinical relevance of metabolically healthy but obese women, in this issue of Diabetologia, Karelis et al. [9] report the original finding that these women may respond differently to an energy-restricted diet than insulin-resistant obese women. From an original population of 121 obese women recruited for a weight loss programme, the authors present data on two selected subgroups of women: those within the upper quartile (the metabolically healthy but obese postmenopausal women) and those within the lower quartile (insulin-resistant and 'at-risk' individuals) of insulin sensitivity values as assessed by means of the euglycaemic-hyperinsulinaemic clamp. The clamp was performed before and after the 
women followed a hypoenergetic diet for 6 months, designed to reduce baseline body mass by $10 \%$, when body weight was stabilised. The dropout rate was $33 \%$ in the metabolically healthy but obese group and $20 \%$ in the obese insulin-resistant group. The two study groups showed comparable reductions in body weight $(6 \%$ and $7 \%$, respectively) and fat mass ( $7 \%$ and $10 \%$, respectively) in response to the diet. The more interesting finding was that, in parallel with the loss of body weight, whole body insulin sensitivity improved (by 26\%) in the obese insulin-resistant women, as expected, whereas it decreased (by 13\%) in the metabolically healthy but obese women.

These results are remarkable because they imply that treating all obese individuals using a nutritional approach might have a detrimental outcome in terms of insulin sensitivity in a considerable proportion (20-30\%) [10]. These preliminary results highlight the need for studies to clarify whether an obese patient should be treated using a dietary approach. That said, there are some issues that should be analysed in more detail.

Taking into consideration both the dropout rates (higher in the metabolically healthy but obese group) and the body fat loss (lower in the metabolically healthy but obese group), the possibility that the metabolically healthy but obese postmenopausal women were more resistant to the diet treatment than the obese insulin-resistant women cannot be completely ruled out.

It should be noted that the plasma insulin concentrations achieved during the clamp procedure in the baseline and post-treatment periods did not closely match. This would be expected in view of the reduction in body weight. As a consequence, when the clamp-derived index of insulin sensitivity was corrected for the real plasma insulin levels, the improvement in insulin sensitivity in the insulin-resistant women was even higher $(+45 \%)$, whereas the detrimental effect in the metabolically healthy but obese postmenopausal women dropped to a non-significant level $(-8 \%)$. According to these values, the difference between these two subgroups in terms of the improvement in insulin sensitivity was even greater, but a truly detrimental effect in the metabolically healthy but obese women was not detectable.

In summary, the study by Karelis et al. confirms that a diet-induced $5-10 \%$ reduction in body weight in obese insulin-resistant women is paralleled by a noteworthy improvement in insulin sensitivity. It also provides evidence that a subgroup of insulin-sensitive obese women may be resistant to dietary treatment, which may even have a small detrimental effect, but this issue requires a more robust demonstration with a more exhaustive analysis. For example, the cardiovascular risk profile of a cohort of metabolically healthy but obese, but in this case premenopausal, women was somewhere between that of non-obese women matched for insulin sensitivity and that of insulin- resistant obese women [11]. Even though blood pressure, HDL-cholesterol, triacylglycerol and fasting insulin values were better in the metabolically healthy but obese premenopausal women than in the insulin-resistant obese subjects, they were still worse than those in the non-obese control women. In particular, intima-media thickness, a marker of cardiovascular disease, was reported to be higher in the metabolically healthy but obese group than in the nonobese control group [11]. As Karelis et al. did not report the cardiovascular risk profile of their metabolically healthy but obese postmenopausal women, it is not possible to conclude whether the energy restriction affected these parameters. From this perspective, it would be interesting to know the outcome of energy restriction not only in those women within the highest (metabolically healthy but obese) and lowest (insulin-resistant) quartiles of insulin sensitivity, but also in those with an intermediate degree of insulin resistance.

A study on insulin-resistant and obese type 2 diabetic patients [12] reported that restriction seemed to have a major effect on hepatic but not skeletal muscle insulin sensitivity. Karelis et al. cannot comment on whether this occurred in their cohort of obese women because they did not perform a glucose tracer infusion during the clamp. Qualitative and quantitative assessment of skeletal muscle and liver insulin sensitivity to determine their relative contributions to the lack of improvement of whole body insulin sensitivity in metabolically healthy but obese postmenopausal women will require additional studies, and a lower insulin infusion rate should be used to explore the hepatic response. If this speculation were to be confirmed, physical exercise may be a more appropriate lifestyle intervention for metabolically healthy but obese individuals. In insulin-resistant or obese individuals it was found that physical training was effective in improving whole body insulin sensitivity (by 16-49\%) [13, 14], but the improvement was not different between insulin-sensitive and insulin-resistant individuals [15]. Some recent indirect evidence suggests that the effectiveness of physical exercise may be inversely related to insulin sensitivity, i.e. exercise does not have a beneficial effect on insulin-resistant muscle [16]. Thus, it is possible that metabolically healthy but obese postmenopausal women would be highly responsive to physical exercise.

Finally, if we postulate a true detrimental effect of a dietary treatment in metabolically healthy but obese postmenopausal women, it will be important to assess whether these findings are reproducible in obese men, in obese premenopausal women and in patients with diabetes. In line with this, and postulating the disadvantage of prescribing an energy-restricted diet for metabolically healthy but obese postmenopausal women, the final crucial issue will be to define how to identify these patients using simple tools. 
In conclusion, the study by Karelis et al. [9] confirms the effectiveness of an energy-restricted diet for improving whole body insulin sensitivity in association with small reductions in body fat. Interestingly, it also shows that there is a subset of individuals, the most insulin sensitive, that is non-responsive to this treatment and may potentially have a detrimental response. This evidence is not sufficient to exclude nutritional support in these individuals at present. Nonetheless, the demonstration of the lack of homogeneous features, and in this study, the lack of homogeneous response to treatments in obese individuals, indicate that we need to apply a better phenotypic characterisation and make greater efforts to tailor the treatment according to the characteristics of the individual patient.

Duality of interest The author declares that there is no duality of interest associated with this manuscript.

\section{References}

1. Bonora E, Kiechl S, Willeit J et al (1998) Prevalence of insulin resistance in metabolic disorders: the Bruneck Study. Diabetes 47:1643-1649

2. Brochu M, Tchernof A, Dionne IJ et al (2001) What are the physical characteristics associated with a normal metabolic profile despite a high level of obesity in postmenopausal women? J Clin Endocrinol Metab 86:1020-1025

3. Meigs JB, Wilson PW, Fox CS et al (2006) Body mass index, metabolic syndrome, and risk of type 2 deiabetes or cardiovascular disease. J Clin Endocrinol Metab 91:2906-2912

4. Perseghin G, Scifo P, Danna M et al (2002) Normal insulin sensitivity and IMCL content in overweight humans are associated with higher fasting lipid oxidation. Am J Physiol Endocrinol Metab 283:E556-E564
5. Karelis AD, Faraj M, Bastard J-P et al (2005) The metabolically healthy but obese individual presents a favourable inflammation profile. J Clin Endocrinol Metab 90:4145-4150

6. Swinburn BA, Nyomba BL, Saad MF et al (1991) Insulin resistance is associated with lower rates of weight gain in Pima Indians. J Clin Invest 88:168-173

7. Ruderman NB, Chisholm D, Pi-Sunyer X, Schneider SH (1998) The metabolically obese, normal-weight individual revisited. Diabetes 47:699-713

8. Dvorak RV, DeNino WF, Ades PA, Poehlam ET (1999) Phenotypic characteristics associated with insulin resistance in metabolically obese but normal-weight young women. Diabetes 48:2210-2214

9. Karelis AD, Messier V, Brochu M, Rabasa-Lhoret R (2008) Metabolically healthy but obese women: effect of an energyrestricted diet. Diabetologia doi:10.1007/s00125-008-1038-4

10. Karelis AD, St-Pierre DH, Conus F, Rabasa-Lhoret R, Poehlman ET (2004) Metabolic and body composition factors in subgroups of obesity: what do we know? J Clin Endocrinol Metab 89:2569-2575

11. Marini MA, Succurro E, Frontoni S et al (2007) Metabolically healthy but obese women have an intermediate cardiovascular risk profile between healthy nonobese women and obese insulinresistant women. Diabetes Care 30:2143-2147

12. Petersen KF, Dufour S, Befroy D, Lehrke M, Hendler RE, Shulman GI (2005) Reversal of nonalcoholic hepatic steatosis, hepatic insulin resistance, and hyperglycaemia by moderate weight reduction in patients with type 2 diabetes. Diabetes 54:603-608

13. Goodpaster BH, Katsiaras A, Kelley DE (2003) Enhanced fat oxidation through physical activity is associated with improvements in insulin sensitivity in obesity. Diabetes 52:2191-2197

14. Gan SK, Kriketos AD, Ellis BA, Thompson CH, Kraegen EW, Chisholm DJ (2003) Changes in aerobic capacity and visceral fat but not myocyte lipid levels predict increased insulin action after exercise in overweight and obese men. Diabetes Care 26:1706-1713

15. Perseghin G, Price TB, Petersen KF et al (1996) Increased glucose transport/phosphorylation and muscle glycogen synthesis after exercise training in insulin-resistant subjects. N Engl J Med 335:1357-1362

16. De Filippis E, Alvarez G, Berria R et al (2008) Insulin-resistant muscle is exercise resistant: evidence for reduced response of nuclear-encoded mitochondrial genes to exercise. Am J Physiol Endocrinol Metab 294:E607-E614 